\title{
Adaptive Control of MEMS Gyroscope Based on Global Terminal Sliding Mode Controller
}

\author{
Weifeng Yan and Juntao Fei \\ Jiangsu Key Lab. of Power Transmission and Distribution Equipment Technology, College of Computer and Information, \\ Hohai University, Changzhou 213022, China
}

Correspondence should be addressed to Juntao Fei; jtfei@yahoo.com

Received 25 June 2013; Accepted 31 October 2013

Academic Editor: Shihua Li

Copyright (C) 2013 W. Yan and J. Fei. This is an open access article distributed under the Creative Commons Attribution License, which permits unrestricted use, distribution, and reproduction in any medium, provided the original work is properly cited.

An adaptive global fast terminal sliding mode control (GFTSM) is proposed for tracking control of Micro-Electro-Mechanical Systems (MEMS) vibratory gyroscopes under unknown model uncertainties and external disturbances. To improve the convergence rate of reaching the sliding surface, a global fast terminal sliding surface is employed which can integrate the advantages of traditional sliding mode control and terminal sliding mode control. It can be guaranteed that sliding surface and equilibrium point can be reached in a shorter finite time from any initial state. In the presence of unknown upper bound of system nonlinearities, an adaptive global fast terminal sliding mode controller is derived to estimate this unknown upper bound. Simulation results demonstrate that the tracking error can be attenuated efficiently and robustness of the control system can be improved with the proposed adaptive global fast terminal sliding mode control.

\section{Introduction}

MEMS gyroscopes are the basic measuring elements of inertial navigation and inertial guidance system, which are commonly used for measuring angular velocity. Because of their enormous advantages in size and cost, MEMS gyroscopes have a wide range of applications in the area of navigation, platform stabilization, control, and traffic. But, due to the manufacturing error and the temperature disturbance, which can cause deviations from the desired qualities and degrade the performance of a gyroscope. Besides, MEMS gyroscopes belong to MIMO systems, which have parameter uncertainties and are vulnerable to external environment influence. Therefore, it is necessary to solve these challenges by utilizing advanced control methods. In the last few years, many advanced control approaches have been proposed to enhance performance and robustness of MEMS gyroscope. Batur et al. [1] developed a sliding mode control for MEMS gyroscope system. Leland [2] presented an adaptive controller for tuning the natural frequency of the drive axis of a vibratory gyroscope. Park et al. $[3,4]$ presented an adaptive controller for a MEMS gyroscope which drives both axes of vibration and controls the entire operation of the gyroscope. Adaptive controller has been developed using sliding mode control to control the vibration of MEMS gyroscope [5]. Raman et al. [6] proposed a closed-loop digitally controlled MEMS gyroscope using unconstrained sigma-delta force balanced feedback control. Tsai and Sue [7] developed integrated model reference adaptive control and time-varying angular rate estimator for microgyroscopes.

As we know, sliding mode control has many attractive features such as robustness to parameter variations and insensitivity to disturbance. The basic idea of the sliding mode control is to drive and maintain the system trajectory on a sliding surface designed a priori in the state space. However, one of the representative characteristics of conventional sliding mode control is that the convergence of the system states to the equilibrium point is usually asymptotical but not in finite time. Recently, a new type of sliding mode control technique called terminal sliding mode control is developed. Instead of using linear hyperplanes as the sliding surfaces, the terminal sliding mode control adopts nonlinear sliding surfaces. With the nonlinear sliding surfaces, the terminal sliding mode controller can make the system states reach the equilibrium point in a finite time 
period. Saif et al. [8] presented a novel methodology for approximation of the unknown time-varying rotation rate by using a sliding mode observer along with a robust control system based on terminal sliding mode control for improving the performance of the MEMS gyroscope. Zhu and Chai [9] presented a global finite-time tracking controller based on terminal sliding mode control method for the trajectory tracking control problem of biped multilinked robots with uncertain disturbances. In order to solve the optimal problem of convergence time and estimate the unknown upper bound of system uncertainties, much research has been done to apply intelligent control approaches such as the adaptive global fast terminal sliding mode control. Feng et al. [10] and Keleher and Stonier [11] proposed an adaptive fast terminal sliding mode tracking control scheme for robotic manipulators to estimate the upper bound of uncertainties. Tao et al. [12] developed a new adaptive fuzzy terminal sliding mode controller for linear systems with mismatched time varying uncertainties. Zhang et al. [13] proposed a new approach of adaptive fuzzy global identical terminal sliding mode control for cross-beam system. Neila and Tarak [14] designed adaptive terminal sliding mode control for rigid robotic manipulators. Feng et al. [15] proposed a nonsingular terminal sliding mode controller for rigid manipulators. In this paper, robust adaptive global fast terminal sliding mode control strategy for trajectory tracking control of MEMS vibratory gyroscopes is proposed. The main motivations in the paper are highlighted as follows.

(1) A global fast terminal sliding mode surface is designed to improve the convergence rate of reaching the sliding surface. It integrates the advantages of traditional sliding and terminal sliding control, and guarantees that the tracking error can converge to zero in a shorter finite time. Adaptive control and terminal sliding control are successfully integrated with MEMS gyroscope.

(2) An adaptive global fast terminal sliding mode control is incorporated into the MEMS gyroscope to improve the tracking performance and strengthen the robustness of the control system. With this control, the upper bound of model uncertainties and external disturbances are not needed because the proposed controller can estimate the upper bound of these uncertainties using an adaptive mechanism. Also, the proposed controller eliminates the chattering effect without losing the robustness property and the precision.

This paper is organized as follows. In Section 2, the dynamics of MEMS gyroscope are described. In Section 3, global fast terminal sliding mode control is introduced. In Section 4, the proposed adaptive global fast terminal sliding mode controller is derived and the stability of this control system is proved by Lyapunov's stability theory. Simulation results are presented in Section 5. Finally, conclusions are provided in Section 6.

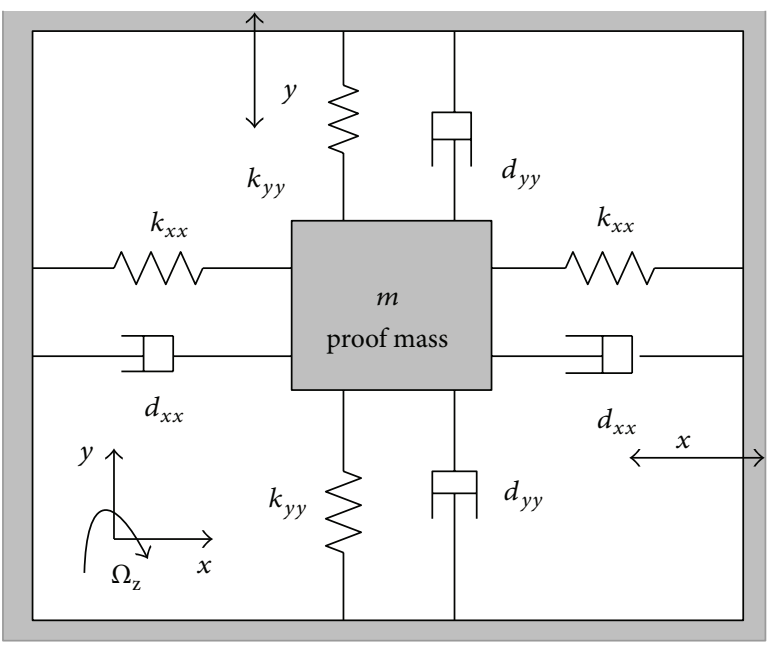

Figure 1: A simplified model of a $z$-axis MEMS Gyroscope.

\section{Dynamics of MEMS Gyroscope}

A $z$-axis MEMS gyroscope is depicted in Figure 1. Generally, a typical MEMS vibratory gyroscope includes a proof mass suspended by spring beams, electrostatic actuations, and sensing mechanisms for forcing an oscillatory motion and sensing the position and velocity of the proof mass. Dynamics of a MEMS gyroscope are derived from Newton's law in the rotating frame.

As we know, Newton's law in the rotating frame becomes

$$
F_{r}=F_{\text {phy }}+F_{\text {centri }}+F_{\text {Coriolis }}+F_{\text {Euler }}=m a_{r} .
$$

In (1), $F_{r}$ is the total applied force to the proof mass in the gyro frame, $F_{\text {phy }}$ is the total physical force to the proof mass in the inertial frame, $F_{\text {centri }}$ is the centrifugal force, $F_{\text {Coriolis }}$ is the Coriolis force, $F_{\text {Euler }}$ is the Euler force, and $a_{r}$ is the acceleration of the proof mass with respect to the gyro frame. $F_{\text {phy }}, F_{\text {Coriolis }}$, and $F_{\text {Euler }}$ are inertial forces caused by the rotation of the gyro frame.

With the definition of $r_{r}$ and $v_{r}$ as the position and velocity vectors relative to the rotating gyroscope frame, and $\Omega$ as the angular velocity vector of the gyroscope frame, the expressions for the inertial forces are reduced to

$$
\begin{gathered}
F_{\text {Coriolis }}=-2 m \Omega \times v_{r}, \quad F_{\text {centri }}=-m \Omega \times\left(\Omega \times r_{r}\right), \\
F_{\text {Euler }}=-m \frac{d \Omega}{d t} \times r_{r},
\end{gathered}
$$

then,

$$
m a_{r}+m \Omega \times\left(\Omega \times r_{r}\right)+2 m \Omega \times v_{r}+m \dot{\Omega} \times r_{r}=F_{\text {phy }},
$$

where $F_{\text {phy }}$ contains spring, damping, and control forces applied to the proof mass.

In a $z$-axis MEMS gyroscope, by supposing the stiffness of spring in $z$ direction much larger than that in $x$ and $y$ directions, motion of proof mass is constrained to only along the $x-y$ plane. Referring to [16], with assuming that the gyroscope is almost rotating at a constant angular velocity 
$\Omega_{z}$ over a sufficiently long time interval, the dynamics of gyroscope based on (3) are simplified as follows:

$$
\begin{aligned}
m \ddot{x}+ & d_{x} \dot{x}+\left[k_{x}-m\left(\Omega_{y}^{2}+\Omega_{z}^{2}\right)\right] x+m \Omega_{x} \Omega_{y} y \\
& =u_{x}+2 m \Omega_{z} \dot{y}, \\
m \ddot{y}+ & d_{y} \dot{y}+\left[k_{y}-m\left(\Omega_{x}^{2}+\Omega_{z}^{2}\right)\right] y+m \Omega_{x} \Omega_{y} x \\
& =u_{y}-2 m \Omega_{z} \dot{x},
\end{aligned}
$$

where $x$ and $y$ are the coordinates of the proof mass with respect to the gyro frame in a Cartesian coordinate system, $d_{x, y}$ and $k_{x, y}$ are damping and spring coefficients, $\Omega_{x, y, z}$ are the angular rate components along each axis of the gyro frame, and $u_{x, y}$ are the control forces in $x$ and $y$ directions. In this design, the control forces are the electrostatic forces in parallel plate actuator which can be expressed as the gradient of the potential energy stored on the capacitor. The last two terms in (4), $2 m \Omega_{z} \dot{y}$ and $2 m \Omega_{z} \dot{x}$ are the Coriolis forces used to reconstruct the unknown input angular rate $\Omega_{z}$. Under typical assumptions $\Omega_{x} \approx \Omega_{y} \approx 0$, only the component of the angular rate $\Omega_{z}$ causes a dynamic coupling between $x$ and $y$-axes.

Taking fabrication imperfections into account, which cause extra coupling between $x$ - and $y$-axes, the lumped parameter mathematical model for an actual $z$-axis MEMS gyroscope becomes

$$
\begin{aligned}
& m \ddot{x}+d_{x x} \dot{x}+d_{x y} \dot{y}+k_{x x} x+k_{x y} y=u_{x}+2 m \Omega_{z} \dot{y}, \\
& m \ddot{y}+d_{x y} \dot{x}+d_{y y} \dot{y}+k_{x y} x+k_{y y} y=u_{y}-2 m \Omega_{z} \dot{x} .
\end{aligned}
$$

In (5), $d_{x x}$ and $d_{y y}$ are damping coefficients, $k_{x x}$ and $k_{y y}$ are spring coefficients, $d_{x y}$ and $k_{x y}$, called quadrature errors, are coupled damping and spring terms, respectively, mainly due to the asymmetries in suspension structure and misalignment of sensors and actuators. The coupled spring and damping terms are unknown, but can be assumed to be small. The nominal values of the $x$-and $y$-axes spring and damping terms are known, but there are small unknown variations. The proof mass can be determined accurately.

Dividing both sides of (5) by $m, q_{0}$, and $\omega_{0}^{2}$, which are a reference mass, length, and natural resonance frequency, respectively, we get the form of the nondimensional equation of motion as

$$
\begin{aligned}
& \ddot{x}+d_{x x} \dot{x}+d_{x y} \dot{y}+\omega_{x}^{2} x+\omega_{x y} y=u_{x}+2 \Omega_{z} \dot{y}, \\
& \ddot{y}+d_{x y} \dot{x}+d_{y y} \dot{y}+\omega_{x y} x+\omega_{y}^{2} y=u_{y}-2 \Omega_{z} \dot{x},
\end{aligned}
$$

where $d_{x x} / m \omega_{0} \rightarrow d_{x x}, d_{x y} / m \omega_{0} \rightarrow d_{x y}, d_{y y} / m \omega_{0} \rightarrow d_{y y}$, $\sqrt{k_{x x} / m \omega_{0}^{2}} \rightarrow \omega_{x}, k_{x y} / m \omega_{0}^{2} \rightarrow \omega_{x y}, \sqrt{k_{y y} / m \omega_{0}^{2}} \rightarrow \omega_{y}$, $\Omega_{z} / \omega_{0} \rightarrow \Omega_{z}$

The vector form of MEMS gyroscope dynamics model can be written as

$$
\ddot{q}+D \dot{q}+K q=u-2 \Omega \dot{q}
$$

where $q=\left[\begin{array}{l}x \\ y\end{array}\right], D=\left[\begin{array}{ll}d_{x x} & d_{x y} \\ d_{x y} & d_{y y}\end{array}\right], K=\left[\begin{array}{cc}\omega_{x}^{2} & \omega_{x y} \\ \omega_{x y} & \omega_{y}^{2}\end{array}\right], u=\left[\begin{array}{l}u_{x} \\ u_{y}\end{array}\right]$, $\Omega=\left[\begin{array}{cc}0 & -\Omega_{z} \\ \Omega_{z} & 0\end{array}\right]$
Considering the system with parametric uncertainties and external disturbance, the dynamics of MEMS gyroscope (7) can be expressed as

$$
\ddot{q}+(D+2 \Omega+\Delta D) \dot{q}+(K+\Delta K) q=u+d,
$$

where $\Delta D$ is the unknown parameter uncertainties of the matrix $D+2 \Omega, \Delta K$ is the unknown parameter uncertainties of the matrix $K$, and $d$ is an uncertain extraneous disturbance.

The control target for MEMS gyroscope is to find a control law so that the proof mass position $\mathbf{q}(t)$ can track the desired trajectory $\mathbf{q}_{r}(t)$. In addition, in the presence of model uncertainties and external disturbances, a robust control design is required to improve the robustness and tracking resolution of the control system. In this paper, adaptive global fast terminal sliding mode control is designed to make the system track the desired trajectory and improve the system robustness in the unknown model uncertainties and external disturbances.

\section{Global Fast Terminal Sliding Mode Control}

The traditional fast terminal sliding mode form can be defined as

$$
s_{a}=\dot{x}_{1}+\beta_{0} x_{1}^{p_{2} / p_{1}}=0,
$$

where $x_{1} \in R^{1}$ is state variable, $\beta_{0}>0$ is constant, and $p_{1}$ and $p_{2}$ are odd positive integers satisfying $p_{1}>p_{2}$. Given an initial state $x_{1}(0) \neq 0$, the system will reach the equilibrium point $x_{1}=0$ along the sliding mode (9) in finite time $t_{c 1}$. The time to reach $x_{1}=0$ from $x_{1}(0) \neq 0$ is determined by

$$
t_{c 1}=\frac{p_{1}}{\beta_{0}\left(p_{1}-p_{2}\right)}\left|x_{1}(0)\right|^{\left(p_{1}-p_{2}\right) / p_{1}} .
$$

The global fast terminal sliding surface can be defined as

$$
s_{b}=\dot{x}_{1}+\alpha_{0} x_{1}+\beta_{0} x_{1}^{p_{2} / p_{1}}=0,
$$

where $\alpha_{0}>0$ is constant. Given an initial state $x_{1}(0) \neq 0$, the system will reach the equilibrium point $x_{1}=0$ along the sliding mode (11) in finite time $t_{c 2}$. The time to reach $x_{1}=0$ from $x_{1}(0) \neq 0$ is determined by

$$
t_{c 2}=\frac{p_{1}}{\alpha_{0}\left(p_{1}-p_{2}\right)} \ln \frac{\alpha_{0} x_{1}(0)^{\left(p_{1}-p_{2}\right) / p_{1}}+\beta_{0}}{\beta_{0}} .
$$

Referring to [17], we can demonstrate that $t_{c 2} \leq t_{c 1}$. Comparing (9) and (11), it can be seen that the convergence times of the two types of terminal sliding modes are all determined by the fast terminal attractor $\dot{x}_{1}=-\beta_{0} x_{1}^{p_{2} / p_{1}}$ when it is far from the equilibrium point. At this time, their convergence rates are basically the same. But when close to the equilibrium point, the convergence time of global fast terminal sliding mode is mainly determined by the linear sliding mode $\dot{x}_{1}=-\alpha_{0} x_{1}$ and the convergence rate accelerates exponentially. At this time, its convergence rate is much better than the traditional fast terminal sliding mode. 


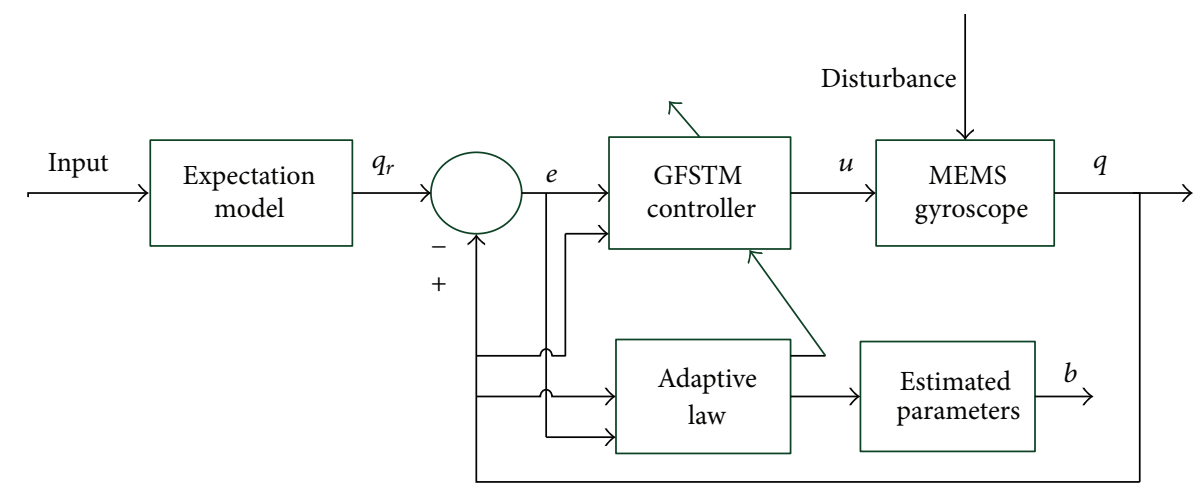

FIGURE 2: Block diagram of an adaptive global fast terminal sliding mode control for a MEMS gyroscope.

Remark 1. The global fast terminal sliding mode not only introduces the fast terminal attractor, making the system state converge in finite time, but also keeps the rapidity of the linear sliding mode when it is close to the equilibrium state, so as to realize the goal of that the system state will converge to the equilibrium state quickly and accurately.

\section{Adaptive Global Fast Terminal Sliding Mode Control}

The global fast terminal sliding mode control that is put forward has solved the optimal problem of convergence time. It integrates the advantages of traditional sliding mode control and terminal sliding mode control in the design of sliding mode and uses the concept of fast access to the equilibrium point at the arriving stage to ensure that the tracking error converges to zero in a shorter finite time. However, as a result of the design principle that is similar to the traditional sliding mode control, there still exists chattering in the global fast terminal sliding mode control signals. Also, the uncertainty boundary needs to be known in advance, and it is hard to accomplish that in actual MEMS gyroscopes. In allusion to the trajectory tracking control of MEMS gyroscopes with unknown model uncertainties and external disturbances, a new adaptive global fast terminal sliding mode control algorithm is synthesized in this section, which uses the respective advantages of adaptive and sliding mode control and the estimates of unknown uncertainty which can be obtained by adaptive control.

The block diagram of an adaptive global fast terminal sliding mode control for a MEMS gyroscope is shown in Figure 2, the tracking error between expectation state and gyroscope state comes to the global fast terminal sliding mode controller. The adaptive global fast terminal sliding mode controller is proposed to control the MEMS gyroscope. The unknown upper bound of system uncertainties can be identified by adaptive estimator.

4.1. Design of Global Fast Terminal Sliding Mode Surface. The tracking control problem of a MEMS gyroscope is what this paper considers, so the control target is to design a suitable control law to make the system output $q$ track the state of the desired trajectory $q_{r}$ completely in finite time.

For the trajectory tracking control of a MEMS gyroscope, two input and two output systems, the global fast terminal sliding surface is chosen as

$$
s_{c}=\left[s_{c 1}, s_{c 2}\right]^{T}=\dot{e}+\alpha e+\beta e^{p_{2} / p_{1}},
$$

where $\alpha=\operatorname{diag}\left(\alpha_{1}, \alpha_{2}\right)$ and $\beta=\operatorname{diag}\left(\beta_{1}, \beta_{2}\right)$ are sliding mode surface constants, $\alpha_{1}, \alpha_{2}, \beta_{1}, \beta_{2}$ are constants, $e=q-$ $q_{r}=\left[q_{1}-q_{r 1}, q_{2}-q_{r 2}\right]^{T}$ is the system tracking error, and $q_{r}$ is the desired trajectory. In the sliding mode $s_{c}=\left[s_{c 1}, s_{c 2}\right]^{T}$, the system reaches the equilibrium state $q=0$ from any initial state $q(0) \neq 0$ in finite time $t_{s}=\left[t_{s 1}, t_{s 2}\right]^{T}$. The time $t_{s}$ is determined by

$$
\begin{aligned}
& t_{s}=\left[\frac{p_{1}}{\alpha_{1}\left(p_{1}-p_{2}\right)} \ln \frac{\alpha_{1} q_{1}(0)^{\left(p_{1}-p_{2}\right) / p_{1}}+\beta_{1}}{\beta_{1}},\right. \\
& \left.\frac{p_{1}}{\alpha_{2}\left(p_{1}-p_{2}\right)} \ln \frac{\alpha_{2} q_{2}(0)^{\left(p_{1}-p_{2}\right) / p_{1}}+\beta_{2}}{\beta_{2}}\right] \text {. }
\end{aligned}
$$

We can make the system reach equilibrium state in finite time $t_{s}$ through setting the suitable parameters $\alpha, \beta, p_{1}$, and $p_{2}$. After determining the sliding surface, the next step is to design a sliding mode controller to ensure the existence of the sliding mode stage.

4.2. Design of Global Fast Terminal Sliding Mode Controller. In the sliding mode control, the control input should be able to force all the system state trajectories to converge the sliding mode surface $s=0$, so as to ensure the existence of the sliding mode stage. The behavior of the sliding mode is equivalent to that of the stability of the state trajectory in the sliding surface. In other words, the control law should be able to ensure that the tracking error converges to zero from any state.

Furthermore, rewriting (8) as

$$
\ddot{q}+(D+2 \Omega) \dot{q}+K q=u+f,
$$

where $f$ represents the matched lumped uncertainty and disturbance which is given by

$$
f=d-\Delta D \dot{q}-\Delta K q
$$


We make the following assumption prior to further discussion.

Assumption 2. The lumped uncertainty and disturbance $f$ is input related and if the control input does not contain the acceleration signal, the system uncertainties will be bounded by a positive function of the position and velocity measurements in the following form:

$$
\|f\|<b_{0}+b_{1}\|q\|+b_{2}\|\dot{q}\|^{2}
$$

where $b_{0}, b_{1}$, and $b_{2}$ are unknown positive constants.

Remark 3. If lumped uncertainty and disturbance $f$ is not related to with state position and velocity signals, the system uncertainty will be only bounded by a positive constant $b_{0}$.

Using the sliding surface (13), the global fast sliding mode controller is proposed as follows:

$$
u=u_{0}+u_{1}+u_{2}
$$

where $u_{0}$ is defined in (19), $u_{1}$ is defined in (20), and $u_{2}$ is defined in (21). Consider

$$
\begin{gathered}
u_{0}=\ddot{q}_{r}+(D+2 \Omega) \dot{q}+K q \\
u_{1}=-\alpha \dot{e}-\frac{p_{2}}{p_{1}} \beta \operatorname{diag}\left(e_{1}^{p_{2} / p_{1}-1}, e_{2}^{p_{2} / p_{1}-1}\right) \dot{e} \\
u_{2}=-\rho \frac{s_{c}}{\left\|s_{c}\right\|} .
\end{gathered}
$$

And $\rho$ has the following expression:

$$
\rho=\xi+b_{0}+b_{1}\|q\|+b_{2}\|\dot{q}\|
$$

where $\xi$ is a positive constant.

According to [18], it is known that the control system is stable in this control law and reaches the stable point in finite time. But the control law requires that $\rho$ should be known. We usually choose the larger $\rho$ conservatively to compensate the system model uncertainties and the upper bound of the disturbances fully, but this often enhances the chattering.

In order to overcome this problem, we introduce a proper adaptation law combining with global fast terminal sliding mode control to realize the adaptive global fast terminal sliding mode control in the next part. This method achieves the goal of tracking system model errors and uncertain disturbances automatically, weakening the chattering and ensuring the stability of the control system.

\subsection{Design of Adaptive Global Fast Terminal Sliding Mode} Controller. Supposing that $\widehat{b}_{0}, \widehat{b}_{1}$, and $\widehat{b}_{2}$ are the estimates of $b_{0}, b_{1}$, and $b_{2}$, respectively, the following simple adaptive control laws can realize the estimation of the unknown parameters $b_{0}, b_{1}$, and $b_{2}$. Consider

$$
\begin{gathered}
\dot{\hat{b}}_{0}=m_{0}\left\|s_{c}\right\|, \\
\dot{\vec{b}}_{1}=m_{1}\left\|s_{c}\right\|\|q\|, \quad \dot{\vec{b}}_{2}=m_{2}\left\|s_{c}\right\|\|\dot{q}\|^{2},
\end{gathered}
$$

where $m_{0}, m_{1}$, and $m_{2}$ are arbitrary positive constants representing the adaptive gains.

Based on the given adaptation law, for the MEMS gyroscope system with the dynamics (15), the adaptive global fast terminal sliding mode controller is designed synthetically as follows, which uses the sliding surface (13):

$$
u=u_{0}+u_{1}+u_{2}
$$

where $u_{0}$ is defined in (25), $u_{1}$ is defined in (26), and $u_{2}$ is defined in (27).

$$
\begin{gathered}
u_{0}=a+(D+2 \Omega) v+K q, \\
u_{1}=-W \frac{s_{c}}{\left\|s_{c}\right\|}, \\
u_{2}=-\frac{s_{c}}{\left\|s_{c}\right\|}\left(\widehat{b}_{0}+\widehat{b}_{1}\|q\|+\widehat{b}_{2}\|\dot{q}\|^{2}\right),
\end{gathered}
$$

where $W=\operatorname{diag}\left(w_{1}, w_{2}\right), w_{i}>0, i=1,2$, is a designed parameter matrix, and

$$
\begin{gathered}
v=\dot{q}_{r}-\alpha e-\beta e^{p_{2} / p_{1}} \\
a=\dot{v}=\ddot{q}_{r}-\alpha \dot{e}-\frac{p_{2}}{p_{1}} \beta \operatorname{diag}\left(e_{1}^{p_{2} / p_{1}-1}, e_{2}^{p_{2} / p_{1}-1}\right) \dot{e}
\end{gathered}
$$

Theorem 4. If the control law (24), with the sliding surface (13) and the adaptation law (23), is applied to the nonlinear uncertain system defined by (15), then the system's tracking error can converge to zero in finite time.

Proof. Let us consider the following positive definite function as a Lyapunov function candidate:

$$
V=\frac{1}{2} s_{c}^{T} s_{c}+\frac{1}{2} \sum_{i=0}^{2} m_{i}^{-1} \widetilde{b}_{i}^{2},
$$

where $\widetilde{b}_{i}=b_{i}-\widehat{b}_{i}$ and $i=0,1,2$ are the estimation errors between actual values and their estimated values, respectively. Since $b_{0}, b_{1}$, and $b_{2}$ are constants, we have $\dot{\widetilde{b}}_{i}=-\dot{\widehat{b}}_{i}$.

Substituting the control $u$ in (24) into the dynamic of MEMS gyroscope (15) yields

$$
\begin{aligned}
\ddot{q}+(D+2 \Omega+\Delta D) \dot{q}+(K+\Delta K) q \\
=a+(D+2 \Omega) v+K q+u_{1}+u_{2}+d .
\end{aligned}
$$

Rewriting (30) yields

$$
\begin{gathered}
(\ddot{q}-a)+(D+2 \Omega)(\dot{q}-v)+\Delta D \dot{q}+\Delta K q \\
=u_{1}+u_{2}+d .
\end{gathered}
$$

Substituting (28) into (31) generates

$$
\dot{s}_{c}+(D+2 \Omega) s_{c}+\Delta D \dot{q}+\Delta K q=u_{1}+u_{2}+d .
$$

Equation (32) can be expressed as

$$
\dot{s}_{c}=-(D+2 \Omega) s_{c}-\Delta D \dot{q}-\Delta K q+u_{1}+u_{2}+d,
$$


from the definition of $f$ in (16), we have

$$
\dot{s}_{c}=-(D+2 \Omega) s_{c}+u_{1}+u_{2}+f .
$$

Differentiating $V$ with respect to time yields, we have

$$
\begin{aligned}
& \dot{V}=s_{c}^{T} \dot{s}_{c}-\sum_{i=0}^{2} m_{i}^{-1} \widetilde{b}_{i} \dot{\widehat{b}}_{i} \\
& =s_{c}^{T}\left[-(D+2 \Omega) s_{c}+u_{1}+u_{2}+f\right]-m_{0}^{-1} \widetilde{b}_{0} \dot{\hat{b}}_{0} \\
& -m_{1}^{-1} \widetilde{b}_{1} \dot{\vec{b}}_{1}-m_{2}^{-1} \widetilde{b}_{2} \dot{\vec{b}}_{2} \\
& =s_{c}^{T}\left(u_{1}+u_{2}+f\right)+s_{c}^{T}[-(D+2 \Omega)] s_{c} \\
& -m_{0}^{-1} \widetilde{b}_{0} \dot{\widehat{b}}_{0}-m_{1}^{-1} \widetilde{b}_{1} \dot{\widehat{b}}_{1}-m_{2}^{-1} \widetilde{b}_{2} \dot{\widehat{b}}_{2} \\
& \leq s_{c}^{T}\left(u_{1}+u_{2}+f\right)-m_{0}^{-1} \widetilde{b}_{0} \dot{\hat{b}}_{0}-m_{1}^{-1} \widetilde{b}_{1} \dot{\hat{b}}_{1}-m_{2}^{-1} \widetilde{b}_{2} \dot{\hat{b}}_{2} \\
& \leq-\lambda_{\min }(W)\left\|s_{c}\right\|+s_{c}^{T}\left(u_{2}+f\right)-m_{0}^{-1} \widetilde{b}_{0} \dot{\hat{b}}_{0} \\
& -m_{1}^{-1} \widetilde{b}_{1} \dot{\hat{b}}_{1}-m_{2}^{-1} \widetilde{b}_{2} \dot{\hat{b}}_{2}=-\lambda_{\min }(W)\left\|s_{c}\right\|+s_{c}^{T} f \\
& -\left\|s_{c}\right\|\left(\widehat{b}_{0}+\widehat{b}_{1}\|q\|+\widehat{b}_{2}\|\dot{q}\|^{2}\right) \\
& -\widetilde{b}_{0}\left\|s_{c}\right\|-\widetilde{b}_{1}\left\|s_{c}\right\|\|q\|-\widetilde{b}_{2}\left\|s_{c}\right\|\|\dot{q}\|^{2} \\
& =-\lambda_{\min }(W)\left\|s_{c}\right\|+s_{c}^{T} f-\left\|s_{c}\right\|\left(b_{0}+b_{1}\|q\|+b_{2}\|\dot{q}\|^{2}\right) \\
& \leq-\lambda_{\min }(W)\left\|s_{c}\right\|+\left\|s_{c}\right\| f \\
& -\left\|s_{c}\right\|\left(b_{0}+b_{1}\|q\|+b_{2}\|\dot{q}\|^{2}\right) \leq-\eta\left\|s_{c}\right\|<0,
\end{aligned}
$$

where $\eta=\lambda_{\min }(W)-\|f\|+\left(b_{0}+b_{1}\|q\|+b_{2}\|\dot{q}\|^{2}\right)>0$ and $\left\|s_{c}\right\| \neq 0[15]$.

Therefore, according to Lyapunov's second method, the designed controller can guarantee the globally asymptotical stability of the system and make the output tracking error converge to zero in finite time. On the other hand, in the global fast terminal sliding mode $s_{c}=\dot{e}+\alpha e+\beta e^{p_{2} / p_{1}}=0$, the output tracking error $e=q-q_{r}$ converges to zero in finite time.

Remark 5. In the proof of Theorem 4, we used the Rayleigh principle, namely,

$$
\lambda_{\min }(W)\|s\|^{2} \leq s^{T} W s \leq \lambda_{\max }(W)\|s\|^{2},
$$

where $\lambda_{\min }(W)$ and $\lambda_{\max }(W)$ are the minimum and maximum eigenvalues of $W$, respectively. Since $W$ is a diagnose matrix, that is, $W=\operatorname{diag}\left[w_{1}, \ldots, w_{n}\right], \lambda_{\min }(W)$ and $\lambda_{\max }(W)$ have the simple types described as follows:

$$
\begin{aligned}
& \lambda_{\text {min }}(W)=\min _{i=1, \ldots, n}\left\{w_{i}\right\}, \\
& \lambda_{\text {max }}(W)=\max _{i=1, \ldots, n}\left\{w_{i}\right\} .
\end{aligned}
$$

Remark 6. Since there are two switching functions sgn in adaptive terminal sliding mode control (26) and (27), there exists chattering in the global fast terminal sliding mode control signals. In order to reduce the chattering, we can use adaptive global fast terminal quasi-sliding mode control instead of adaptive global fast terminal sliding mode control. The so-called adaptive global fast terminal quasi-sliding mode control is a control method that the system trajectory is confined to a neighborhood $\delta$ within the ideal sliding mode. The quasi-sliding mode control adopts normal sliding mode control in the boundary layer outside and continuous state feedback control in the boundary layer, so as to avoid or weaken the chattering. Therefore, the controller (24) is improved as follows:

$$
\begin{gathered}
u_{1}= \begin{cases}-W \frac{s_{c}}{\left\|s_{c}\right\|}, & \text { if }\left\|s_{c}\right\|>\delta, \\
-W \frac{s_{c}}{\delta}, & \text { if }\left\|s_{c}\right\| \leq \delta,\end{cases} \\
u_{2}= \begin{cases}-\frac{s_{c}}{\left\|s_{c}\right\|}\left(\widehat{b}_{0}+\widehat{b}_{1}\|q\|+\widehat{b}_{2}\|\dot{q}\|^{2}\right), & \text { if }\left\|s_{c}\right\|>\delta, \\
-\frac{s_{c}}{\delta}\left(\widehat{b}_{0}+\widehat{b}_{1}\|q\|+\widehat{b}_{2}\|\dot{q}\|^{2}\right), & \text { if }\left\|s_{c}\right\| \leq \delta,\end{cases}
\end{gathered}
$$

where $\delta$ is a designed positive parameter; then, the chattering will be eliminated.

\section{Simulation Example}

In this section, we will evaluate the proposed adaptive global fast terminal sliding mode control scheme on the lumped MEMS gyroscope sensor model by using Matlab/Simulink. The control objective is to design an adaptive global fast terminal sliding mode controller so that the trajectory $q(t)$ can track the state of the desired model $q_{r}(t)$ completely in finite time. In addition, the unpredictably upper bound of system uncertainties can be estimated by adaptive estimator. The parameters of the MEMS gyroscope are as follows:

$$
\begin{gathered}
m=1.8 \times 10^{-7} \mathrm{~kg}, \quad k_{x x}=63.955 \mathrm{~N} / \mathrm{m}, \\
k_{y y}=95.92 \mathrm{~N} / \mathrm{m}, \quad k_{x y}=12.779 \mathrm{~N} / \mathrm{m}, \\
d_{x x}=1.8 \times 10^{-6} \mathrm{Ns} / \mathrm{m}, \quad d_{y y}=1.8 \times 10^{-6} \mathrm{Ns} / \mathrm{m}, \\
d_{x y}=3.6 \times 10^{-7} \mathrm{Ns} / \mathrm{m} .
\end{gathered}
$$

Since the general displacement range of the MEMS gyroscope in each axis is sub-micrometer level, it is reasonable to choose $1 \mu \mathrm{m}$ as the reference length $q_{0}$. Given that the usual natural frequency of each axis of a MEMS gyroscope is in the $\mathrm{KHz}$ range, so, choose the $\omega_{0}$ as $1 \mathrm{KHz}$. The unknown angular velocity is assumed $\Omega_{z}=100 \mathrm{rad} / \mathrm{s}$. Then, the nondimensional values of the MEMS gyroscope parameters are listed as follows:

$$
\begin{gathered}
\omega_{x}^{2}=355.3, \quad \omega_{y}^{2}=532.9, \\
\omega_{x y}=70.99, \quad d_{x x}=0.01, \\
d_{y y}=0.01, \quad d_{x y}=0.002, \quad \Omega_{z}=0.1 .
\end{gathered}
$$




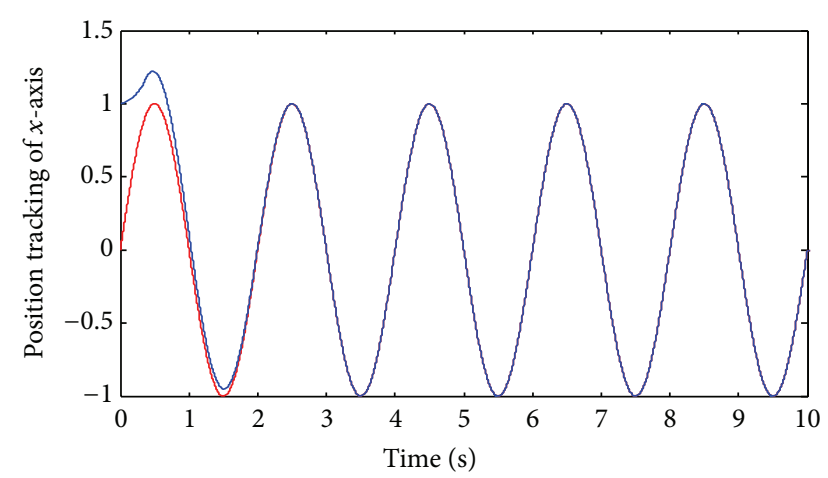

- Desired trajectory of $x$-axis

- Actual trajectory of $x$-axis

FIgURE 3: Position tracking of $x$-axis.

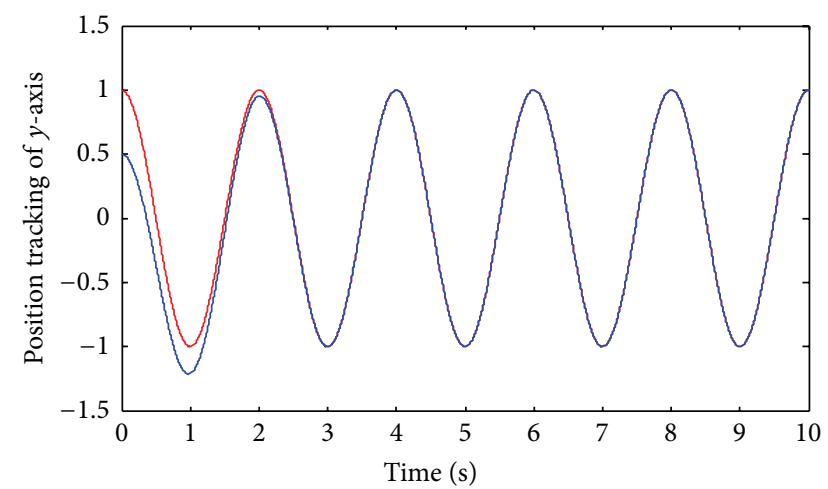

— Desired trajectory of $y$-axis

- Actual trajectory of $y$-axis

FIgURE 4: Position tracking of $y$-axis.

In this simulation example, the initial values of the system are selected as $q_{1}(0)=1.0, \dot{q}_{1}(0)=0, q_{2}(0)=$ 0.5 , and $\dot{q}_{2}(0)=0$. The desired motion trajectories are described by $q_{r 1}=\sin (\pi t)$ and $q_{r 2}=\cos (\pi t)$. The lumped parametric uncertainties and external disturbances given by $f=d-\Delta D \dot{q}-\Delta K q$ are composed of model uncertainties and external disturbances. As for model uncertainties, there are $\pm 10 \%$ parameter variations for the spring and damping coefficients with respect to their nominal values and $\pm 10 \%$ magnitude changes in the coupling terms, that is, $d_{x y}$ and $\omega_{x y}$ with respect to their nominal values. Random signal $d=[0.5 * \operatorname{randn}(1,1) ; 0.5 * \operatorname{randn}(1,1)]$ is considered as external disturbances. The chosen sliding surface parameters are $p_{1}=5, p_{2}=3, \alpha_{1}=\alpha_{2}=0.25$, and $\beta_{1}=\beta_{2}=0.5$ and the initial conditions of the upper bound of the uncertainty are $b_{00}=0.1, b_{10}=0.2$, and $b_{20}=0.3$. The adaptive gains are chosen as $m_{0}=1.0, m_{1}=1.6$, and $m_{2}=1.6$. The sliding mode controller parameter of $(26)$ is $W=\operatorname{diag}(2,4)\left(w_{1}=\right.$ $\left.2, w_{2}=4\right)$. In order to have a small boundary layer around the sliding surface and reduce the chattering, we have chosen the designed positive parameter $\delta$ equal to 0.06 and used the adaptive global fast terminal quasi-sliding mode control (36). The simulation results are shown in Figures 3-9.

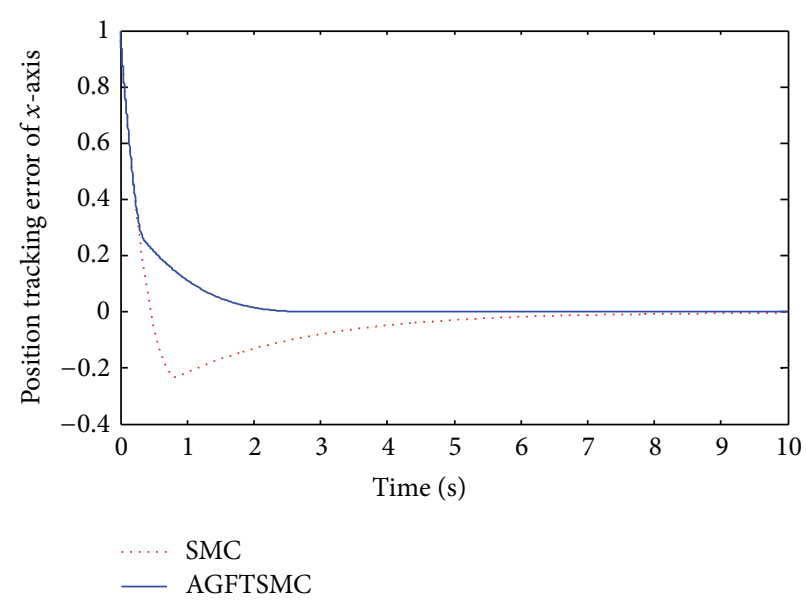

FIGURE 5: Position tracking error of $x$-axis comparison between AGFTSMC and SMC.

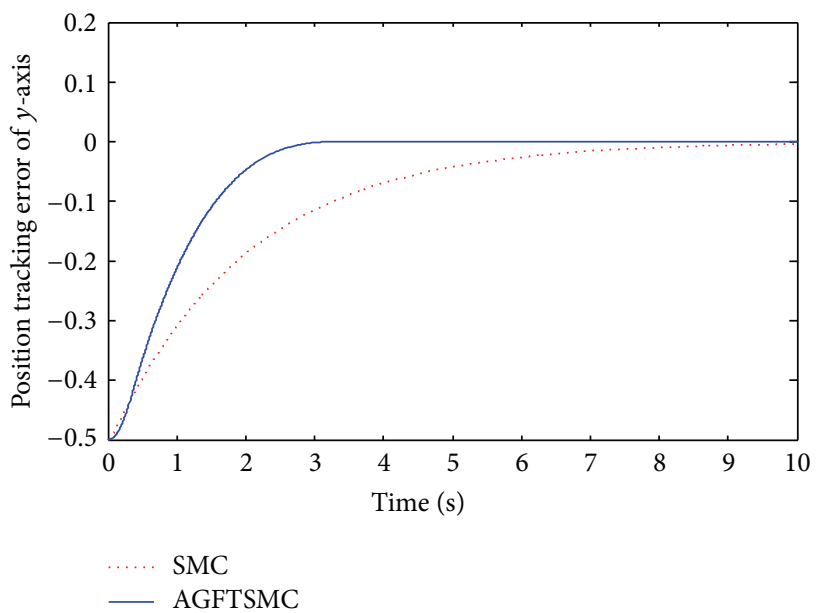

FIGURE 6: Position tracking error of $y$-axis comparison between AGFTSMC and SMC.

Figures 3 and 4 depict the MEMS gyroscope along $x$ axis and $y$-axis tracking trajectories using the adaptive global fast terminal sliding mode control law, respectively. From Figures 3 and 4 , it can be observed intuitively that after about 3 seconds, the actual motion trajectory of the MEMS gyroscope is consistent with the desired reference trajectory, making the system state track the state of the desired trajectory completely in a shorter finite time.

Subsequently, in order to demonstrate the advantages of the paper, a comparable investigation on MEMS gyroscope model (15) is accomplished between the proposed adaptive global fast terminal sliding mode control and conventional sliding mode control, and the results are shown in Figures 5 and 6 , where the tracking errors of $x$-axis and $y$-axis corresponding to adaptive global fast terminal sliding mode control more severely decrease in contrast with the conventional sliding mode control due to the proposed finite time convergence algorithm. Besides, from the Figures 5 and 6, it can be also seen that the tracking errors of adaptive global 

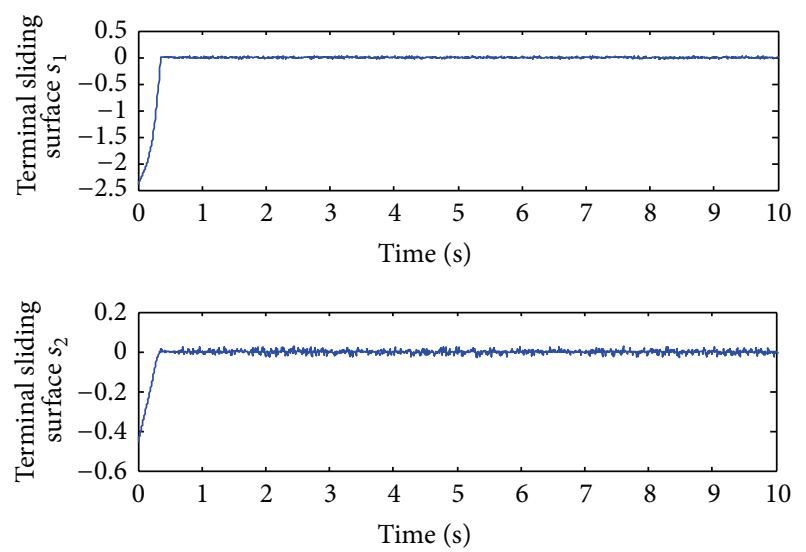

FIGURE 7: Convergence of the terminal sliding surface $s(t)$.

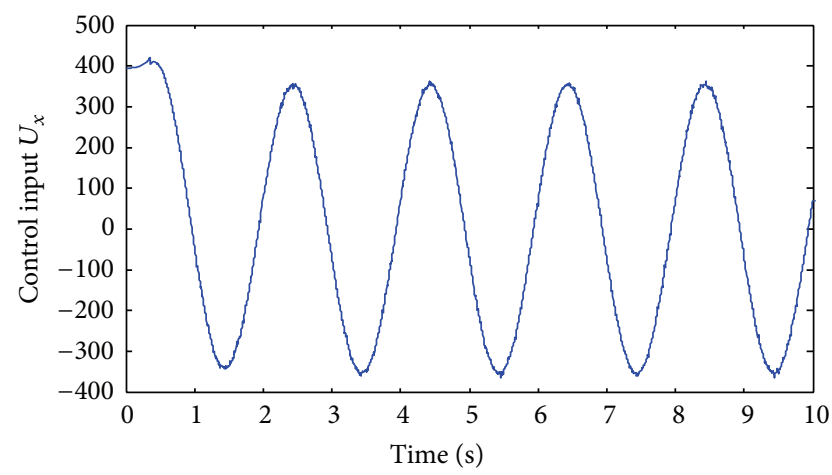

FIGURE 8: Control input signal of $x$-axis.

fast terminal sliding mode control converge to zero in about 3 seconds, which improves the dynamic behavior of the MEMS gyroscope and verify that the adaptive global fast terminal sliding mode control can ensure the global stability of the control system.

Moreover, we can set the appropriate sliding mode surface parameters to adjust the convergence rate, so as to improve the work efficiency. Figure 7 describes the convergence of the global fast terminal sliding mode surface $s(t)$. It is shown that the sliding mode surface converges to zero in a very short time. The global fast terminal sliding mode control has solved the optimal problem of convergence time compared with the traditional terminal sliding mode so that it can make the system state converge to the equilibrium state quickly and accurately. It should be noted that we can also choose appropriate reaching law to improve the convergence rate further, such as ideal reaching law and adaptive reaching law, which have been proposed in recent years. Sliding mode control system utilized these two types of reaching laws that not only can reach the region of the switching surface in finite time but also enhance the dynamic performance of the control system.

Figures 8 and 9 depict the smooth sliding mode control forces of the MEMS gyroscope along $x$-axis and $y$-axis with the adaptive global fast terminal sliding mode controller, respectively. It is clearly observed from Figures 8 and 9 that there is no chattering using the proposed controller. Figure 10

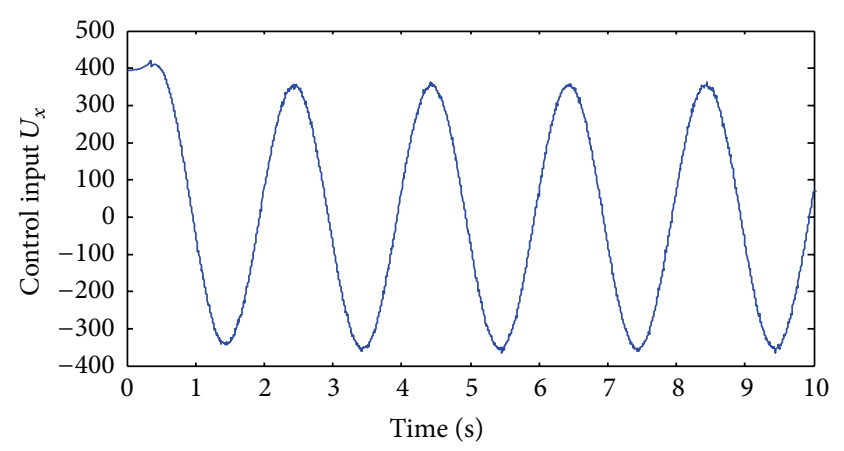

FIgURE 9: Control input signal of $y$-axis.
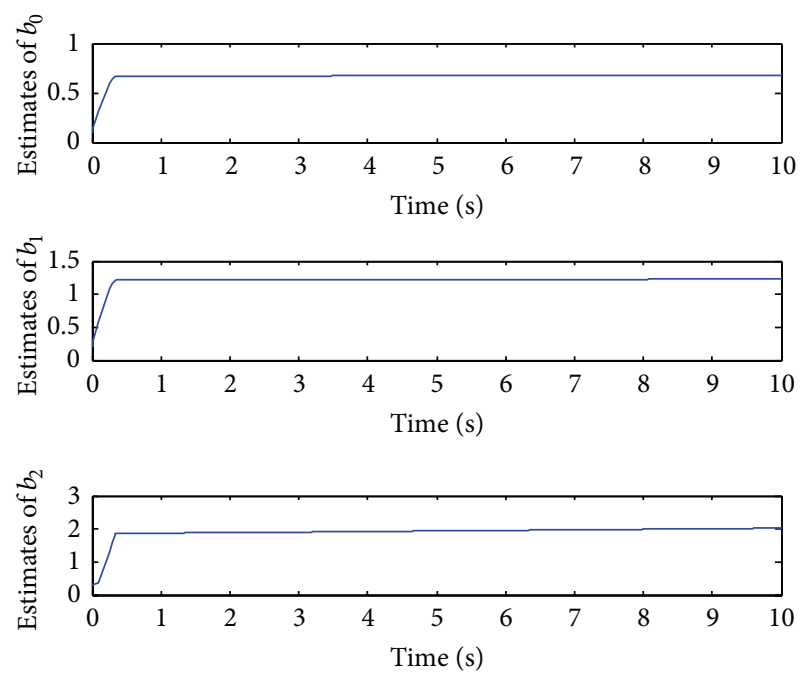

FIgURE 10: Adaptively estimated parameters $\widehat{b}_{0}, \widehat{b}_{1}$, and $\widehat{b}_{2}$.

presents the adaptively estimated parameters $\widehat{b}_{0}, \widehat{b}_{1}$, and $\widehat{b}_{2}$. These results show a stable convergence of the upper bound parameters.

In the presence of large uncertainties, the global fast terminal sliding mode control can give large tracking error which is not the case in adaptive global fast terminal sliding mode control. Therefore, the introduction of adaptive control technique can adapt to the changes of external environment and control structure parameters, which maintains the optimal performance of the control system. In addition, adaptive technology can effectively solve the robustness problem in the presence of unknown model parameters and external disturbances, so as to improve the MEMS gyroscope's reliability and disturbance rejection ability.

\section{Conclusion}

In this paper, we proposed the design of a new adaptive global fast terminal sliding mode controller for trajectory tracking control of MEMS vibratory gyroscopes, which enables the robustness in the presence of parameter uncertainties and the external disturbances. The main feature of this design is that it combines the global fast terminal sliding mode control with a boundary layer and the adaptive approach. 
Based on the Lyapunov's stability theory, the system state with the designed global fast terminal sliding mode controller is able to converge to equilibrium point in a shorter finite time. In order to reduce the chattering, we present a softening control approach. Considering the unknown upper bound of system uncertainties, an adaptive global fast terminal sliding mode controller with unpredictably upper bound of parameter estimation is derived. The adaptive algorithm is used to estimate the bounds of uncertainties and external disturbances. Simulation results are provided to demonstrate the validity and reliability of the proposed control scheme.

\section{Acknowledgments}

The authors thank the anonymous reviewers for their useful comments that improved the quality of the paper. This work is partially supported by the National Science Foundation of China under Grant no. 61374100, the Natural Science Foundation of Jiangsu Province under Grant no. BK20131136, and the Fundamental Research Funds for the Central Universities under Grant no. 2012B06714.

\section{References}

[1] C. Batur, T. Sreeramreddy, and Q. Khasawneh, "Sliding mode control of a simulated MEMS gyroscope," ISA Transactions, vol. 45, no. 1, pp. 99-108, 2006.

[2] R. P. Leland, "Adaptive control of a MEMS gyroscope using lyapunov methods," IEEE Transactions on Control Systems Technology, vol. 14, no. 2, pp. 278-283, 2006.

[3] S. Park, R. Horowitz, S. K. Hong, and Y. Nam, "Trajectoryswitching algorithm for a MEMS gyroscope," IEEE Transactions on Instrumentation and Measurement, vol. 56, no. 6, pp. 25612569, 2007.

[4] S. Park and R. Horowitz, "New adaptive mode of operation for MEMS gyroscopes," Journal of Dynamic Systems, Measurement and Control, vol. 126, no. 4, pp. 800-810, 2004.

[5] J. Fei and C. Batur, "A novel adaptive sliding mode control with application to MEMS gyroscope," ISA Transactions, vol. 48, no. 1, pp. 73-78, 2009.

[6] J. Raman, E. Cretu, P. Rombouts, and L. Weyten, "A closedloop digitally controlled MEMS gyroscope with unconstrained sigma-delta force-feedback," IEEE Sensors Journal, vol. 9, no. 3, pp. 297-305, 2009.

[7] N.-C. Tsai and C.-Y. Sue, "Integrated model reference adaptive control and time-varying angular rate estimation for micromachined gyroscopes," International Journal of Control, vol. 83, no. 2, pp. 246-256, 2010.

[8] M. Saif, B. Ebrahimi, and M. Vali, "Terminal sliding mode control of Z-axis MEMS gyroscope with observer based rotation rate estimation," in Proceedings of the American Control Conference (ACC '11), pp. 3483-3489, July 2011.

[9] D. Zhu and Y. Chai, "Tracking control of multi-linked robots based on terminal sliding mode," in Proceedings of the 30th Chinese Control Conference (CCC '11), pp. 2539-2543, July 2011.

[10] Y. Feng, X. Yu, and Z. Man, "Adaptive fast terminal sliding mode tracking control of robotic manipulator," in Proceedings of the 40th IEEE Conference on Decision and Control (CDC '01), pp. 4021-4026, December 2001.
[11] P. G. Keleher and R. J. Stonier, "Adaptive terminal sliding mode control of rigid robotic manipulator with uncertain dynamic incorporating constraint inequalities," ANZIAM Journal, vol. 43, pp. 102-153, 2001.

[12] C. W. Tao, J. S. Taur, and M.-L. Chan, "Adaptive fuzzy terminal sliding mode controller for linear systems with mismatched time-varying uncertainties," IEEE Transactions on Systems, Man, and Cybernetics, Part B, vol. 34, no. 1, pp. 255-262, 2004.

[13] J. Y. Zhang, W. J. Gu, Z. D. Liu, and R. P. Chen, "Adaptive fuzzy global identical terminal sliding mode control for cross-beam system," in International Conference on Systems and Informatics, pp. 51-55, 2012.

[14] M. B. R. Neila and D. Tarak, "Adaptive terminal sliding mode control for rigid robotic manipulators," International Journal of Automation and Computing, vol. 8, no. 2, pp. 215-220, 2011.

[15] Y. Feng, X. Yu, and Z. Man, "Non-singular terminal sliding mode control of rigid manipulators," Automatica, vol. 38, no. 12, pp. 2159-2167, 2002.

[16] S. Park, Adaptive control strategies for MEMS gyroscope [Ph.D. dissertation], University of California, Berkeley, Calif, USA, 2000.

[17] K.-B. Park and T. Tsuji, "Terminal sliding mode control of second-order nonlinear uncertain systems," International Journal of Robust and Nonlinear Control, vol. 9, no. 11, pp. 769-780, 1999.

[18] S. Yu, X. Yu, and Z. Man, "Robust global terminal sliding mode control of SISO nonlinear uncertain systems," in Proceedings of the 39th IEEE Confernce on Decision and Control, pp. 2198-2203, December 2000. 


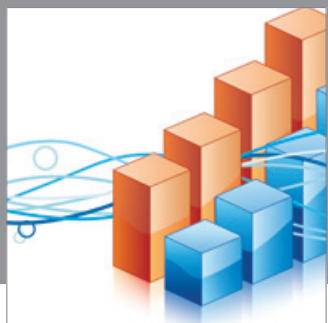

Advances in

Operations Research

mansans

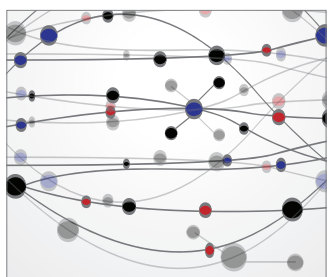

The Scientific World Journal
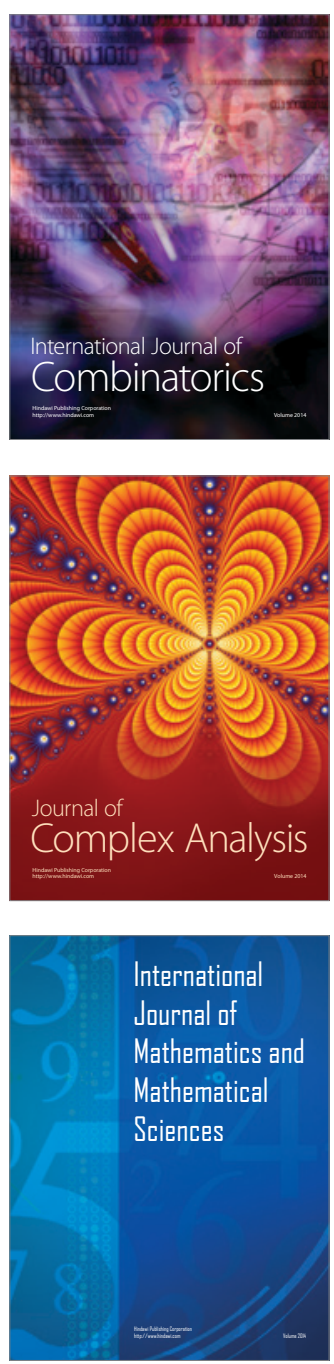
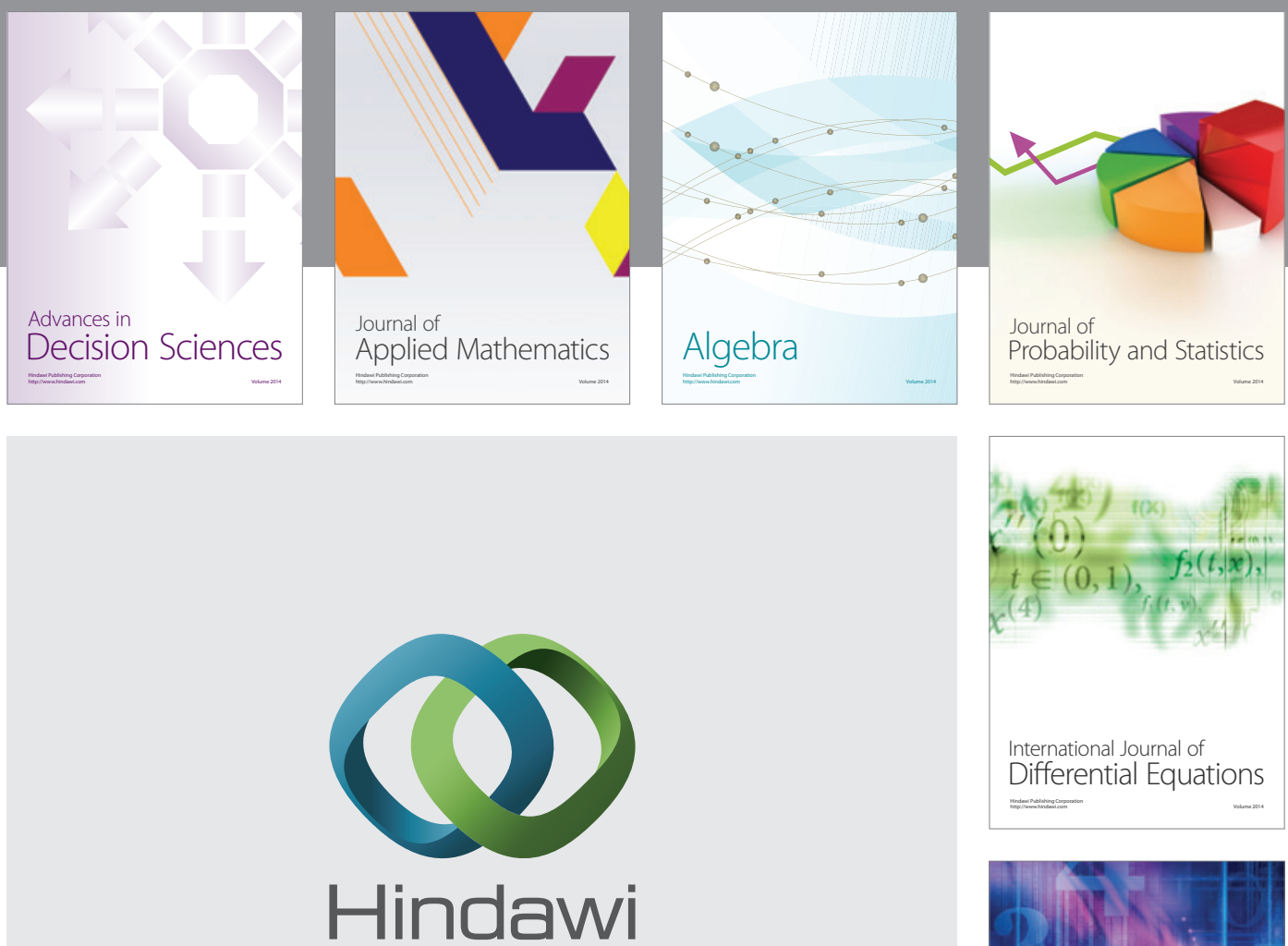

Submit your manuscripts at http://www.hindawi.com
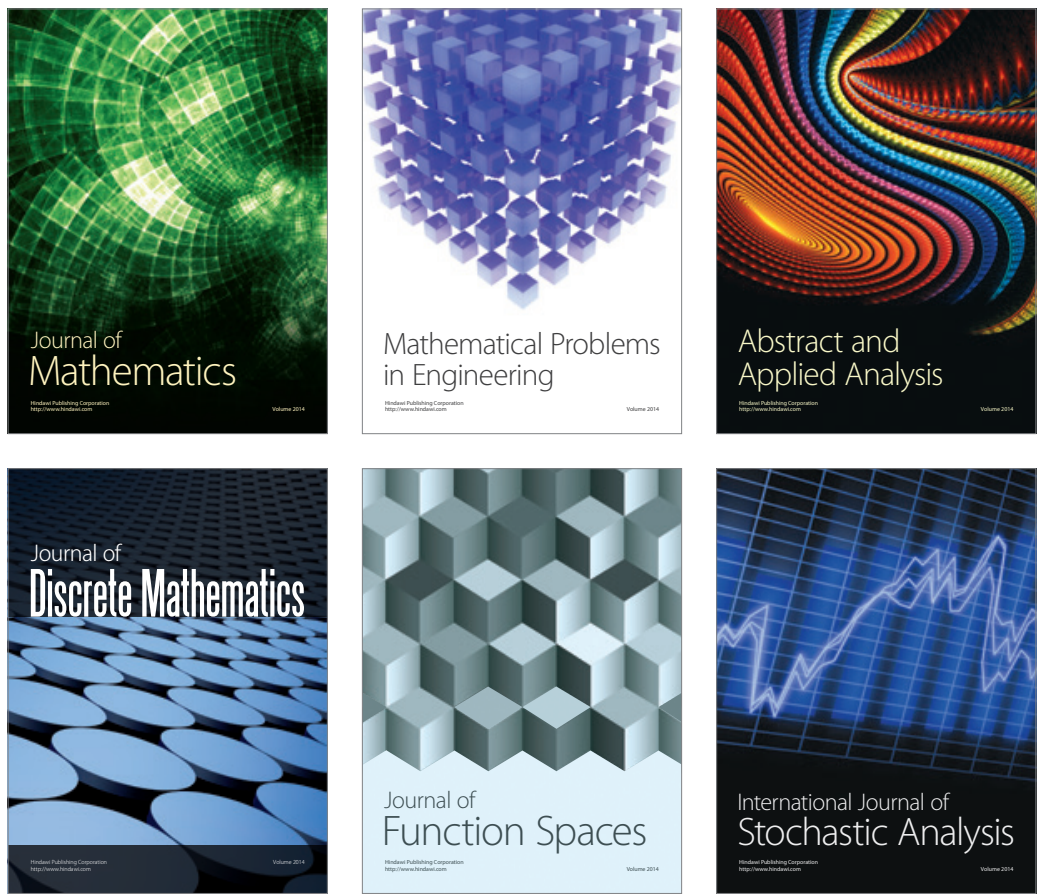

Journal of

Function Spaces

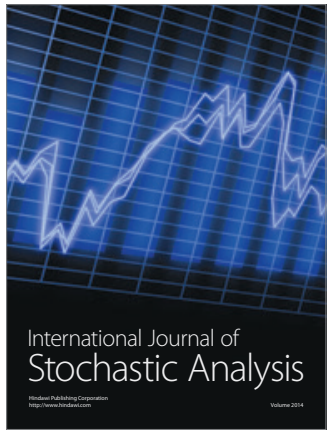

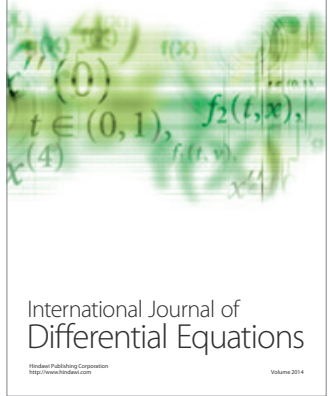
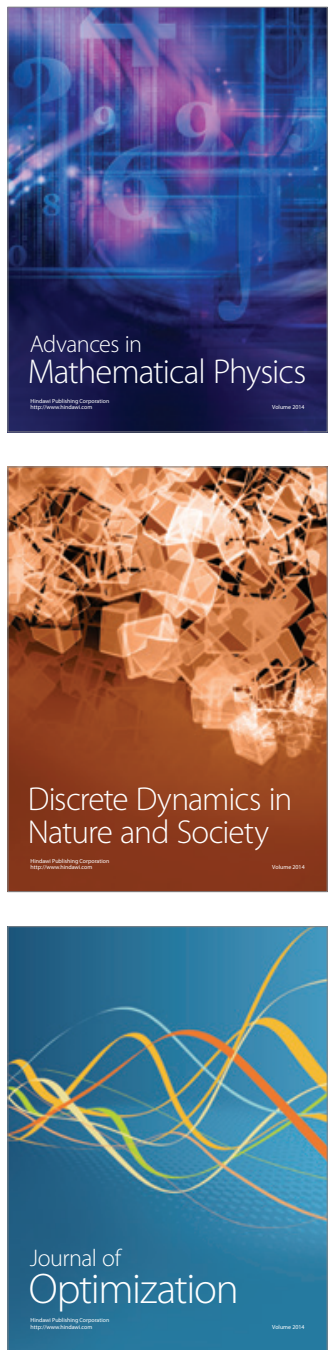